\title{
LA NORMATIVA JURÍDICA EN ROMA A TRAVÉS DE LOS CONSULADOS DE P. CORNELIO ESCIPIÓN EMILIANO
}

\author{
ARCADIO DEL CASTILLO \\ Universidad de Alicante
}

\begin{abstract}
Sobre la base de la existencia de la excepción en la legislación romana, hecho siempre posible, pasamos a delimitar los problemas que se plantean cuando se analizan las elecciones por las que fue entregado a P. Cornelio Escipión Emiliano el consulado en los años 147 y 134 a.C.

This paper concentrates on the permanent possibility, under Roman Law, of adducing exceptions to the norms, as an approach to the outline of the problems arising from the analysis of the elections which led to the appointment of P. Cornelius Scipio Aemilianus as consul in 147 and 134 B.C.
\end{abstract}

Existe una tendencia en la legislación romana durante el Imperio que mantiene la curiosa costumbre de establecer en toda norma jurídica la posibilidad de que, por indulgencia del emperador, la excepción a la regla era siempre factible. Durante la época republicana, sin embargo, la excepción no podía ser potestad de una única persona, pero ello no implica que no fuese también posible; $y$, a veces, de una manera incluso más que curiosa. Tal sucede en el caso que aquí nos ocupa: los dos nombramientos consulares de P. Cornelio Escipión Emiliano.
Tras el fracaso del cónsul P. Calpurnio Pisón Cesonino durante sus operaciones contra Cartago en el año 148 a.C., en Roma cundió la ansiedad ante una guerra que se tornaba difícil, por lo que el pueblo deseaba vivamente que P. Cornelio Escipión Emiliano fuese enviado al teatro de operaciones, en la confianza de que él llevaría la guerra a un final favorable para los intereses romanos.

De esta manera, en el 147 a.C., según nos comunica Apiano, el referido personaje participaba en las elecciones como candidato a la edilidad, ya que las leyes 
no le permitían el acceso al consulado a causa de su edad; sin embargo, el pueblo lo eligió cónsul, lo que era ilegal, y, cuando los cónsules mostraron al pueblo la ley, éste gritó que, por las leyes de Tulio y Rómulo, él era el juez de las elecciones y podía legalmente confirmar o rechazar lo que quisiese; por ello, uno de los tribunos de la plebe declaró que retiraría a los cónsules el poder de celebrar una elección si no cedían a las pretensiones del pueblo, razón por la que entonces el Senado permitió a los tribunos de la plebe suspender esa ley y ponerla en vigor después de un año:

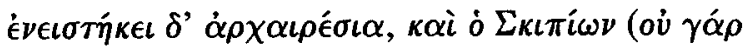

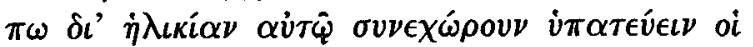

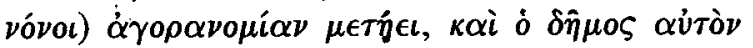

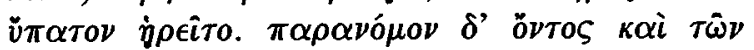

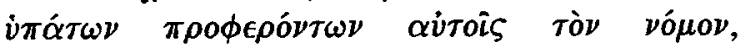

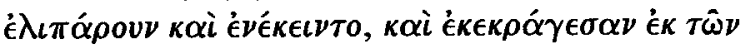

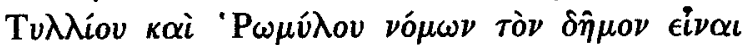

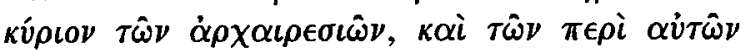

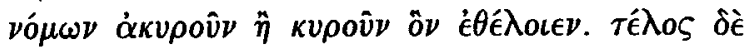

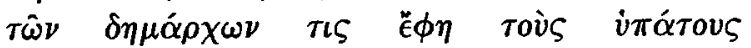

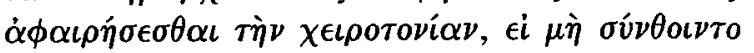

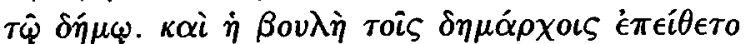

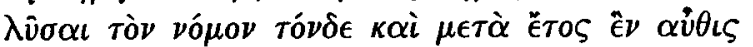

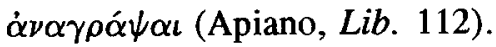

Tito Livio incide en la misma idea de que se presentó a la edilidad siendo elegido cónsul por el pueblo; y que, como no tenía la edad necesaria para serlo, el pueblo protestó y le apoyó, por lo que, pese a una cierta oposición por parte de los senadores, se le eximió de las leyes y fue nombrado cónsul: P. Scipio Aemilianus cum aedilitatem peteret, consul a populo dictus. Quoniam per annos consuli fieri non licebat, cum magno certamine suffragantis plebis et repugnantibus ei aliquamdiu patribus, legibus solutus et consul creatus (Liv., Per. 50).

De una forma más escueta, Cicerón dice que nunca pidió el consulado, pero que fue hecho cónsul dos veces, la primera antes de tiempo, esto es antes de la edad correspondiente: ...qui consulatum petiit nunquam, factus est consul bis, primum ante tempus... (Cic., Amic. 3, 11); lo mismo aparece en la Rhetorica ad Herennium: ... ante tempus consulem fieri (Rhet. Her. 3, 2); Diodoro Sículo que el pueblo hizo que se le concediese el consulado aunque no se lo permitían ni su edad ni las leyes:

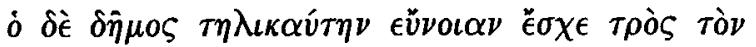

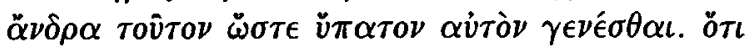

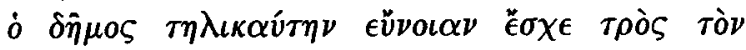

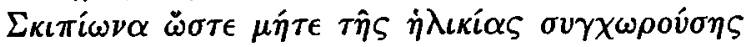

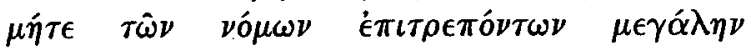

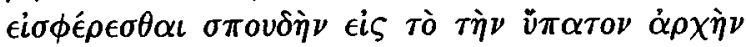
$\alpha \dot{v} \tau \bar{\varphi} \pi \epsilon \rho \iota \theta \epsilon \hat{\imath} \nu \alpha \iota$ (Diod., 32, 9a, 2-3);

el de uiris illustribus que solicitó la edilidad, pero que se le otorgó el consulado antes de tener la edad necesaria: Cum aedilitatem peteret, consul ante annos ultro factus... (de uir. ill. 58, 5); Eutropio que se le hizo cónsul siendo aún joven: ...iuuenis adhuc consul est factus... (Eutr., 4, 12, 1); y Zonaras que recibió el consulado, aunque su edad no le autorizaba para ejercer el cargo:

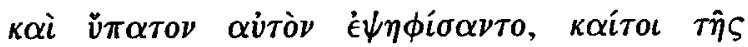
$\dot{\eta} \lambda \iota \kappa i \alpha \varsigma \mu \grave{\eta} \dot{\epsilon} \phi \iota \epsilon i \sigma \eta \varsigma \alpha \dot{v} \tau \bar{\varphi} \tau \grave{\eta} \nu \dot{\alpha} \rho \chi \bar{\eta} \nu$ (Zon., 9, 29).

También nuevamente Apiano vuelve a decir que le concedieron el consulado siendo joven (Apiano, B. C. 3,88 ). Respecto a una cita de Plutarco, en la que, hablando de que C. Mario fue elegido cónsul por segunda vez contra la ley que impedía designar un ausente y también reelegir magistrado a alguien sin que se guardase el espacio de tiempo prefijado, dice que se consideró que no era la primera vez que la ley cedía al interés del Estado y que la razón de entonces no era menor que la que había hecho que P. Cornelio Escipión Emiliano fuese igualmente elegido cónsul contra la ley, puesto que no temían la ruina de Roma, sino que deseaban solamente destruir Cartago:

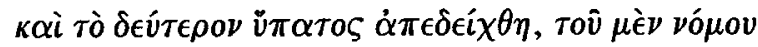

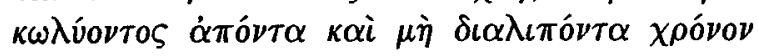

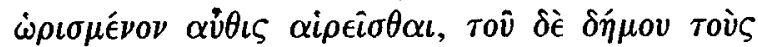

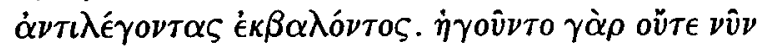

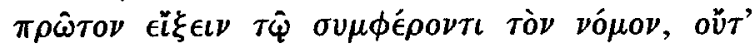

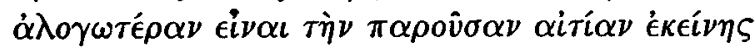

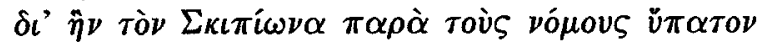
$\dot{\alpha} \pi \epsilon \in \delta \epsilon \iota \xi \alpha \nu, \quad o \dot{v} \quad \phi o \beta o v ́ \mu \epsilon \nu o \iota \quad \tau \grave{\eta} \nu \quad \dot{\epsilon} \alpha v \tau \hat{\omega} \nu$ $\dot{\alpha} \pi \rho \beta \alpha \lambda \epsilon \bar{\imath} \nu, \quad \dot{\alpha} \lambda \lambda \dot{\alpha} \quad \tau \grave{\eta} \nu \quad \mathrm{K} \alpha \rho \chi \eta \delta \delta \nu i \omega \nu$ $\dot{\epsilon} \pi \iota \theta v \mu o \hat{\nu} \nu \tau \epsilon \varsigma \dot{\alpha} \nu \epsilon \lambda \epsilon \hat{\imath} \nu$ (Plut., Mar. 12, 1-2).

No creemos que esta referencia se pueda interpretar, según pretende T.R.S. Broughton, como una equivocación de Plutarco y que este autor aluda a la reelección del ano 134 a.C. (BROUGHTON, I, pág. 491 nota 1), puesto que se trata de una comparación 
en términos generales. Por lo demás, Veleyo Patérculo manifiesta únicamente que fue elegido cónsul, aunque se presentó como candidato a la edilidad: Ita eodem tempore P. Scipio Aemilianus....aedilitatem petens consul creatus est (Vel. Pat., 1, 12, 3); e igualmente, Valerio Máximo que el pueblo le hizo cónsul, pese a haberse presentado como candidato para ser edil: Aemilianum enim populus ex candidato aedilitatis consulem fecit (Val. Max., 8, 15, 4). Existen otras referencias menos relevantes, que solamente denotan que fue elegido cónsul (cfr. Cic., Phil. 11, 7, 17; Mur. 28, 58; Aul. Gel., N.A. 16, 8, 10; Apiano, B.C. 1, 19 y 4, 92).

La elección se produjo mediante el plebiscitum de lege soluendo P. Cornelio Scipione (cfr. ROTONDI, págs. 293-294). Por lo que respecta al precepto legal que le impedía la elección como cónsul, se trataba de la lex Villia annalis del año 180 a.C. (cfr. MOMMSEN, I, págs. 529-530 y 537; ROTONDI, págs. 278-279; BROUGHTON, I, pág. 388), un plebiscito del tribuno de la plebe L. Vilio, que marcaba la edad en que podía aspirarse a las diferentes magistraturas y tomar posesión de ellas. Así lo denotan autores como Tito Livio: Eo anno rogatio primum lata est ab L. Villio tribuno plebis, quot annos nati quemque magistratum peterent caperentque (Liv., 40, 44, 1); Cicerón: ... aeuitatem annali lege seruando (Cic., Leg. 3, 3, 9); Ovidio:... finitaque certis legibus est aetas, unde petatur honor... (Ovid., Fasti 5, 65); o Festo: Annaria lex dicebatur ab antiquis ea, qua finiuntur anni magistratus capiendi (Fest., Epit. p. 27). Cicerón, haciendo referencia a esta normativa, expresa que se fijó una edad bastante madura para el consulado: Legibus enim annalibus cum grandiorem aetatem ad consulatum constituebant... (Cic., Phil. 5, 17, 47). Y este mismo autor da a entender que, en su época, la edad mínima requerida para este cargo era de cuarenta y dos años: Quid ? Macedo Alexander, cum ab ineunte aetate res maximas gerere coepisset, nonne tertio et tricesimo anno mortem obiit? quae est aetas nostris legibus decem annis minor quam consularis (Cic, Phil. 5, 17, 48). Naturalmente la alusión de Cicerón al consulado diez años después, lo colocaba en su cuadragésimo tercer año; y ello además se ve apoyado por el consulado del mismo Cicerón, que, habiendo nacido en el 106, fue cónsul en el 63 a.C., su cuadragésimo tercer año, aunque al comenzar el año del consulado tenía cuarenta y dos, esto es el mínimo exigido, tal como pone de manifiesto él mismo al decir que llegó al consulado suo anno (Cic., Brut. 94, 323; Leg. Agr. 2, 2, 3-4; Off. 2, 17, 59. Cfr. MOMM-
SEN, I, págs. 527 nota 1 y 566 nota 1; FRACCARO, págs. 225 y 228 ; ASTIN, págs. 31 y 32 nota 1 ). Th. Mommsen ha mantenido la misma posición con posterioridad a la legislación de Sila, pero no necesariamente en la lex Villia, la cual habría propugnado que, salvo dispensa especial, podía revestirse el consulado encontrándose en el año trigésimo cuarto o en el trigésimo séptimo, éste en el caso de haber ocupado la edilidad curul, e igualmente en los años trigésimo primero y trigésimo cuarto respecto a la pretura (MOMMSEN, I, págs. 565-568); el planteamiento de este autor viene dado por su suposición de que la lex Villia habría fijado la edad mínima para aspirar a la magistratura primera, esto es la cuestura, la cual, habida cuenta de que se necesitaba la prestación de un servicio militar de diez años o haber estado inscrito por el mismo tiempo en la lista del ejército, sería de veintiocho años, y los intervalos bianuales necesarios producirían las edades ya referidas. Sin embargo, existen autores que defienden la idea de que la lex Villia estableció la edad mínima para cada una de las magistraturas (cfr. MARTINO, II, pág. 415, así como la pág. 416 nota 7 , donde especifica la abundante bibliograffa existente, tanto de una como de otra posición). G. de Sanctis acepta las edades mínimas propuestas por Th. Mommsen, aunque considera que fueron aumentadas ya en la primera mitad del siglo II a.C., seguramente por la lex Pinaria annalis (SANCTIS, IV-1, pág. 497 nota 60. Supone también que esta ley era posterior a la Villia, NIPPERDEY, pág. 6. En contra, MARTINO, II, pág. 414. Cfr. la discusión sobre el particular en FRACCARO, pág. 208 nota 7 y ASTIN, págs. 41-42 nota 3). En cualquier caso, E.A. Astin ha puesto de manifiesto, con sólidos argumentos, que en el período posterior a Sila la edad mínima para el consulado era de cuarenta y dos años, y probablemente de treinta y nueve para la pretura, y treinta y seis para la edilidad curul (ASTIN, págs. 33-34), y que las mismas edades se requerían para las magistraturas curules en la época anterior, por lo que hay que suponer que ese mismo sistema fue el establecido por la lex Villia (ASTIN, pág. 41). Pues bien, como consecuencia de los hechos ocurridos, P. Cornelio Escipión Emiliano llegaría a su primer consulado, al comenzar el 147 a.C., cuando aún tenía sólo treinta y siete años, habida cuenta de que hubo de nacer en el 185 o todo lo más a principios del 184 a.C. (cfr. ASTIN, pág. 34).

Al mismo tiempo, le fue asignada, sin el correspondiente sorteo, la provincia de Africa, mediante la lex de prouincia P. Cornelio Scipioni extra sortem 
danda (cfr. ROTONDI, pág. 294). En tal sentido se manifiesta Apiano, que dice que, cuando su colega en el consulado, C. Livio Druso, le pidió que se hiciese el sorteo para designar quien tendría Africa como su provincia, uno de los tribunos de la plebe propuso que la designación de este mandato fuese hecha por el pueblo, y éste eligió a P. Cornelio Escipión Emiliano:

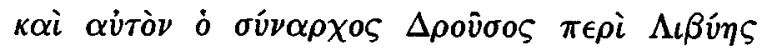

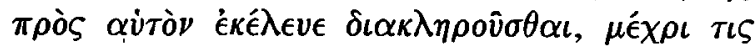
$\tau \bar{\omega} \nu \delta \eta \mu \alpha \dot{\alpha} \rho \omega \nu \dot{\epsilon} \sigma \eta \gamma \dot{\eta} \sigma \alpha \tau 0 \tau \hat{\eta} \sigma \delta \epsilon \tau \hat{\eta} \varsigma \sigma \tau \rho \alpha \tau \eta \gamma \dot{i} \alpha \varsigma$

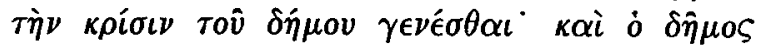

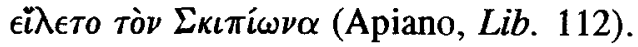

Tito Livio, en cambio, únicamente expresa que se le había dado la provincia de Africa directamente, sin mediar el sorteo: ....deinde a Scipione consule, cui extra sortem Africa prouincia data erat (Liv., Per. 51). Y lo mismo denota Valerio Máximo, aunque especificando que fue el Senado quien le confirió la provincia: Eidem senatus bis sine sorte prouinciam, prius Africam, deinde Hispaniam dedit... (Val. Max., 8, 15, 4).

En el año 134 a. C. la guerra de Numancia se había convertido para los romanos en un auténtico problema. Tras los éxitos de Q. Cecilio Metelo Macedónico en los años 143 y 142 a.C. esta ciudad vino a convertirse en un auténtico baluarte de los celtíberos, y su lucha llenó de vergüenza a Roma, que vio como sus sucesivos gobernadores hubieron de retirarse ante el empuje de una pequeña ciudad, en la que el número de combatientes no superaba los ocho mil hombres. El hecho aparece claramente constatado especiálmente en los fracasos de Q. Pompeyo en los años 141 y 140, que incluso llegó a acordar un pacto con los numantinos a espaldas del Senado, en el nuevo descalabro de M. Popilio Lena en el 138, o en la catástrofe de C. Hostilio Mancino, que, después de ser repetidamente derrotado por los numantinos, siendo finalmente cercado en el campamento romano cercano a Renieblas, tomó la decisión de firmar un tratado que ligaba a romanos y numantinos como iguales, y ello pese a disponer de un contingente armado de unas cinco veces superior a aquel al que se enfrentaba; todo ello sumado a que los gobernadores del 136 y $135, \mathrm{~L}$. Furio Filón y Q. Calpurnio Pisón, no se atrevieron de ninguna manera a atacar Numancia (cfr. en general, CASTILLO, págs. 277 ss.).

El Senado necesitaba ahora acabar rápidamente con la situación que se había creado, porque, como comenta Apiano, en Roma, el pueblo estaba ya cansado de esta guerra, que se alargaba y les resultaba mucho más difícil de lo que esperaban (Apiano, $I b$. 84). Ahora bien, tampoco estaban dispuestos los romanos a aceptar la vergüenza de un tratado con los numantinos. Por esta razón, debido al cariz que habían tomado los acontecimientos, se necesitaba un hombre de grandes dotes, muy experimentado en los combates. Solamente P. Cornelio Escipión Emiliano parecía ser la persona idónea para acabar con el problema numantino. Tomada la decisión era necesario elegirlo para el consulado. De esta manera, P. Cornelio Escipión Emiliano fue elegido para desempeñar su segundo consulado. Sin embargo, en esta elección existieron ciertos problemas jurídicos que es necesario analizar.

Apiano manifiesta que el pueblo romano se encontraba ya cansado de la guerra numantina, que era más larga y difícil de lo esperado -como ya hemos denotado anteriormente-, por lo que eligió a P. Cornelio Escipión Emiliano, el destructor de Cartago, para desempeñar de nuevo el consulado, en la creencia de que era el único capaz de vencer a los numantinos, y que, puesto que también ahora tenía menos edad de la establecida por la ley para acceder al consulado, el Senado, tal como se había hecho en su primer consulado para luchar contra los cartagineses, decretó que los tribunos de la plebe dejaran en suspenso la ley referente al límite de edad y la pusiesen nuevamente en vigor al año siguiente:

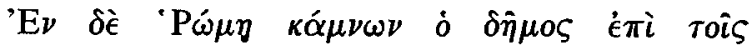

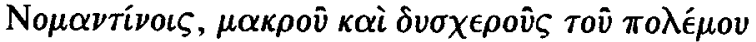

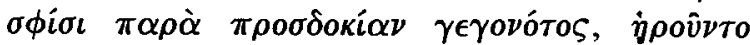

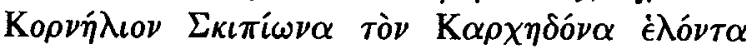
$\alpha \dot{v} \theta \iota \varsigma \dot{v} \pi \alpha \tau \epsilon \dot{v} \epsilon \iota \nu, \dot{\omega} \varsigma \mu o_{\nu o \nu} \epsilon \dot{\epsilon} \iota \rho \alpha \tau \hat{\eta} \sigma \alpha \iota \tau \hat{\omega} \nu$

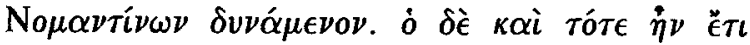

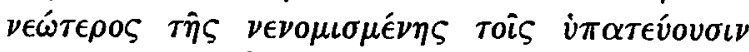
$\dot{\eta} \lambda \iota \kappa i \alpha \varsigma^{\circ} \dot{\eta}$ oiv $\beta 0 v \lambda \grave{\eta} \pi \dot{\alpha} \lambda \iota \nu, \quad \check{\omega} \sigma \pi \epsilon \rho \quad \dot{\epsilon} \pi i$

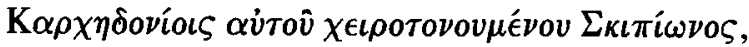

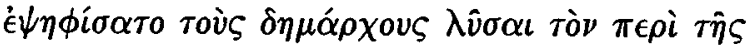

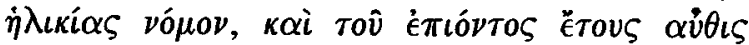
$\theta \dot{\epsilon} \sigma \theta \alpha \iota$ (Apiano, Ib., 84).

Tito Livio, en cambio, nos comunica que, como la guerra de Numancia se alargaba con falta de jefes $y$ no sin vergüenza pública, el Senado y el pueblo le ofrecieron el consulado y que, no siéndole permitido ser cónsul por la ley que prohibía la reelección, fue dispensado de las restricciones legales, como había 
sucedido con su primer consulado: Cum bellum Numantinum uitio ducum non sine pudore publico duraret, delatus est ultro Scipioni Africano a senatu populoque Romano consulatus; quem cum illi capere ob legem, quae uetabat quemquam iterum consulem fieri, non liceret, sicut priori consulatu legibus solutus est (Liv., Per. 56). Por lo demás, Valerio Máximo sugiere únicamente que había sido elegido cónsul por segunda vez en un momento en que había ido para apoyar con su voto a su sobrino durante una elección de cuestores: Eundem, cum quaestoriis comitiis suffragator $Q$. Fabi Maximi, fratris filii, in campum descendisset, consulem iterum reduxit (Val. Max., 8, 15, 4); y Cicerón que no era candidato, pero que la elección se hizo a su tiempo: ...qui consulatum petiit nunquam, factus est consul bis...., iterum sibi suo tempore... (Cic., Amic. 3, 11) y en ausencia: ...deligere iterum consul absens... (Cic., Rep. 6, 11, 11). Otros autores simplemente hacen referencia a la elección sin más (cfr. Cic., Diu. Caec. 21, 69; Mur. 28, 58; Vel. Pat., 2, 4, 2; Apiano, B.C. 1, 19; Plut., Mor. 201A; Eutr., 4, 17, 2; Oros., 5, 7, 1).

Existe obviamente una importante diferencia en lo expuesto por los dos autores que más pormenorizadamente nos hablan de esta elección, esto es, Apiano y Tito Livio. Ambos coinciden en manifestar que existía un precepto legal que le impedía ser reelegido cónsul y que tal impedimento fue resuelto de la misma manera como había ocurrido respecto a su elección para el primer consulado. Pero, ahí se acaba toda coincidencia, puesto que mientras el primero indica que fue dispensado de la ley que prohibía la reelección, el segundo considera que se realizó una suspensión provisional, durante un año, de la ley que decretaba la edad mínima para acceder al consulado. ¿Cuál pudo ser la verdadera razón que hizo necesario recurrir a un procedimiento especial para saltarse lo anteriormente legislado? ¿Cuál era la ley que impedía la reelección de P. Cornelio Escipión Emiliano para el consulado? Analicemos pormenorizadamente la situación.

Si hacemos caso a Apiano, el problema tendría que ver también en esta ocasión con la lex Villia annalis. Por supuesto, como hemos explicado ya antes, es cierto que en el 147 a.C. el Senado decretó que los tribunos de la plebe suspendiesen por aquel año esta ley, debido a que entonces P. Cornelio Escipión Emiliano no tenía la edad requerida para el consulado. Sin embargo, no se puede olvidar que P.
Cornelio Escipión Emiliano, al comenzar el 134 a.C., tenía ya cincuenta años, con lo que había sobrepasado ampliamente la edad mínima requerida. Por eso tiene sentido lo expresado por Cicerón de que la elección se hizo a su tiempo.

Si nos atenemos a lo manifestado por Tito Livio habría que pensar en una ley que impidiese a $\mathrm{P}$. Cornelio Escipión Emiliano simplemente ser reelegido. J. Arce ha interpretado que se trataba de la norma que prohibía el ejercicio de una magistratura, en este caso el consulado, dos veces en el espacio de diez años: «La legislación del año 151, que prohibía a una misma persona ser cónsul dos veces en el espacio de diez años, era el obstáculo legal que impedía que Escipión pudiera acceder al mando del ejército consular, hasta que no transcurriese el tiempo reglamentario» (ARCE, pág. 91). Aunque este autor hace referencia a que tal norma era del 151 a.C., lo cierto es que el contenido tiene que ver con el plebiscito del año 342 a. C., que Tito Livio parece atribuir al tribuno de la plebe L. Genucio (Liv., 7, 42, 1-2. Cfr. ROTONDI, págs. 224-225; BROUGHTON, I, pág. 134), que Th. Mommsen tiende a considerarlo de época posterior, en concreto del 330 a.C. (MOMMSEN, I, pág. 520 nota $5)$, y que pretendía impedir el monopolio de los cargos, mediante la imposición de que existiese un intervalo de diez años para poder asumir la misma magistratura (Liv., 7, 42, 2; Cic., Leg. 3, 3, 9; Zon., 7, 25). Ahora bien, tal norma no resulta aplicable en el caso que estamos tratando, puesto que entre el primer consulado de P. Cornelio Escipión Emiliano en el año 147 a.C. y el segundo en el 134 a.C. median más de los referidos diez años.

No obstante, no hemos de suponer que Tito Livio estuviera equivocado, ya que no dice expresamente que se refiera a la ley que prohibía la reelección en el intervalo de diez años; si así fuese, lo hubiese concretado. Este autor hace referencia solamente a una norma jurídica que prohibía la reelección. Pues bien, M. Claudio Marcelo fue reelegido cónsul por tercera vez, en el año 152 a.C., porque el Senado, ante el desastre de Q. Fulvio Nobílior, consideró necesario recurrir a una persona como él, de gran experiencia en la guerra. Para poder hacerlo fue necesario autorizar mediante ley su reelección, pese a no haber transcurrido el período necesario de diez años para poder ejercer la misma magistratura como imponía el plebiscito del año 342 a.C.: M. Claudio Marcelo había sido cónsul primeramente en el 166 a.C. y después 
igualmente en el 155 a.C. Todo parece inclinar a pensar que esta reelección fue posiblemente la que provocó la lex de consulatu non iterando del 151 a.C., probablemente un plebiscito, apoyado por M. Porcio Catón, que estableció que nadie pudiera ser reelegido para el consulado (cfr. MOMMSEN, I, pág. 521 nota 1; ROTONDI, págs. 290-291). Esta norma fue abrogada en el 82 a.C., mediante la lex Cornelia de magistratibus (cfr. MOMMSEN, I, pág. 521; ROTONDI, pág. 351), a la vez que, como apunta Apiano, se restablecía la del 342 a.C. (Apiano, B. C. $1,100)$, razón por la que, cuando Cicerón se refiere a las limitaciones en el ejercicio de las magistraturas, dice que se debía de observar la edad límite fijada y que no se podía ejercer la misma magistratura excepto después de un intervalo de diez años (Cic., Leg. 3, $3,9)$, no expresando que en el caso del consulado la simple reelección estaba prohibida. En estas condiciones, parece fuera de toda posible duda que Tito Livio habría de referirse a esta norma jurídica del 151 a.C., con lo que el Senado permitió a P. Cornelio Escipión Emiliano ejercer por segunda vez el consulado en el 134 a.C., decretando que los tribunos de la plebe dejaran en suspenso por ese año precisamente la referida norma (un nuevo plebiscitum de lege soluendo P. Cornelio Scipione. Cfr. ROTONDI, pág. 298; BROUGHTON, I, pág. 490). Que, ante una necesidad perentoria, se pueda admitir la excepción en una ley de carácter general, parece admisible; que tal hecho se repita con demasiada frecuencia ya lo es menos; pero, no puede dejar de resultar ciertamente bastante curioso que se establezca una ley para impedir la reelección en el consulado, que tal hecho fuese el resultado de un caso en el que la ley había sido transgredida mediante un precepto jurídico expreso, y todo ello para que esa nueva ley establecida volviera a ser transgredida de manera similar (el hecho se repitió con los consulados de C. Mario en los años 104-100 y 86 a.C., de L. Cornelio Cinna en 86-84 a.C., y de Cn. Papirio Carbón en 84 y 82 a.C., cfr. BROUGHTON, I, págs. 558-574, y II, págs. 53-60 y 65). Incluso, se da el agravante, en la mayoría de los casos, de que, además de la existencia de la prohibición para la reelección, tampoco se hubiese cumplido el precepto de la espera de un intervalo de diez años).

Por lo demás, asimismo en este momento, le fue asignada sin sorteo la provincia de Hispania Citerior, seguramente también en esta ocasión mediante una ley, igual que en el año 147 a.C., aunque Valerio
Máximo atribuye también tal designación al Senado (Val. Max., 8, 15, 4. Cfr. ROTONDI, pág. 298).

En definitiva, todo el proceso que rodea a los dos consulados de P. Cornelio Escipión Emiliano se enmarca dentro de un esquema que resulta corriente en el sistema legislativo romano, en el que muy a menudo la excepción a la norma jurídica era siempre posible cuando ello se consideraba necesario. Y cuando se daba el caso, la norma se transgredía de una forma también legal, de manera que la excepción se suponía entonces perfectamente correcta.

\section{BIBLIOGRAFÍA}

ARCE, J., «Las guerras celtíbero-lusitanas», en J.M. Roldán y otros, Historia de España Antigua, Il. Hispania Romana, $2^{a}$ ed., Madrid, 1985, págs. 79-98.

ASTIN, E.A., The Lex Annalis before Sulla (Coll. Latomus, vol. XXXII), Bruxelles, 1958.

BROUGHTON, T.R.S., The Magistrates of the Roman Republic, III, Cleveland, 1951.

CASTILLO. A. del, «La conquista romana de Hispania: lusitanos y celtíberos», en J.M. Blázquez y A. del Castillo, Manual de Historia de España, 1. Prehistoria y Edad Antigua, Madrid, 1991, págs. 263-291

FRACCARO, P., «I «decem stipendia» e le «leges annales» repubblicane», Opuscula, II, Pavia, 1957, págs. 207-234.

MARTINO, F. de, Storia della costituzione romana, II, $2^{\mathrm{a}} \mathrm{ed}$,, Napoli, 1973.

MOMMSEN, Th., Römisches Staatsrecht, I, reimp. Graz, 1969.

NIPPERDEY, K., «Die Leges annales der römischen Republik», Abhandlungen der philologisch-historischen Classe der Königlich Sächsischen Gesellschaft der Wissenschaften, V (Abhandlungen der Königlich Sächsischen Gesellschaft der Wissenschaften, XII), 1870, págs. 1-88.

ROTONDI, G., Leges publicae populi romani, reimp. Hildesheim, 1966.

SANCTIS, G. de, Storia dei romani, IV- 1, $2^{a}$ ed., Firenze, 1969 